\title{
Die Dialektisch-Behaviorale Therapie bei der Borderline-Persönlichkeitsstörung
}

Christian Stiglmayr

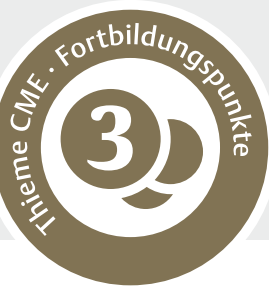

\section{Die gestörte Emotionsregulation der Borderline-Störung wird in der DBT mit einer Kombination aus akzeptanz- und veränderungsbasierten Strategien bei gleich- zeitiger Berücksichtigung wesentlicher therapeutischer Grundannahmen behan- delt. Die DBT orientiert sich hierbei dynamisch an den vom Patienten gezeigten Verhaltensmustern.}

\section{Einleitung}

Die Dialektisch-Behaviorale Therapie (DBT) wurde in den 80er-Jahren von Prof. Marsha M. Linehan zur Behandlung von Patientinnen und Patienten mit einer Borderline-Persönlichkeitsstörung (BPS) entwickelt [1, 2]. Linehan konzipierte die Therapie ursprünglich als ambulante Behandlung von chronisch suizidalen Frauen. Heute gilt die DBT als Prototyp für modular aufgebaute Psychotherapie und findet bei einer Vielzahl weiterer Störungsbilder Anwendung. Neben den etablierten kognitiv-behavioralen Methoden integriert die DBT eine Vielzahl weiterer Strategien und Techniken, v. a. aus den Bereichen:

- Gesprächspsychotherapie

- Gestalttherapie

- Hypnotherapie

- Zen-Buddhismus

Damit gehört die DBT zur sog. „dritten Welle“ von Verhaltenstherapie, welche die klassischen verhaltenstherapeutischen und kognitiven Techniken mit Strategien der Akzeptanz, Weisheit, kognitiven Distanzierung und Dialektik verbindet.

\section{THERAPIESÄULEN}

Die DBT ruht auf 2 Säulen: die eine Säule symbolisiert die zur Anwendung kommenden Techniken/Strategien; die andere Säule symbolisiert die Haltung des Therapeuten. Eine klare Struktur gibt den Säulen Halt und Stabilität.

\section{Therapiestruktur}

\author{
Merke \\ Im Verständnis der DBT handelt es sich bei der BPS \\ um eine Emotionsregulationsstörung, bedingt durch
}

ein Zusammenspiel von ungünstigen biologischen wie auch sozialen Einflüssen.

Ätiologische Basis. Auf Grundlage eines biosozialen Ätiologiemodells wird davon ausgegangen, dass zum einen eine hohe Sensitivität für emotionale Reize besteht. Durchschnittlich zeigen sich 9-mal stärkere emotionale Reaktionen sowie eine deutlich verlangsamte Rückkehr auf das emotionale Ausgangsniveau (z.B. [3]). Zum anderen haben die Patienten unzureichende Fertigkeiten, mit dieser erhöhten emotionalen Vulnerabilität funktional umzugehen. Die Folge ist, dass die Patienten ihre Emotionen als unkontrollierbar erleben und zwar sowohl unangenehme Emotionen wie Scham, Schuld, Ärger, Angst wie auch angenehme Emotionen wie Liebe, Freude oder Stolz [4]. Gleichzeitig lernen die Patienten aufgrund eines invalidierenden Umfeldes keine funktionalen Strategien im Umgang mit diesen starken Emotionen. Ein invalidierendes soziales Umfeld ist durch die Tendenz gekennzeichnet, unangemessen und unberechenbar auf persönliche Erfahrungen des Kindes zu reagieren. Dem Kind wird vermittelt, dass es das, was es angibt zu fühlen, gar nicht fühle (z.B. „wenn sie nein sagt, meint sie eigentlich ja“) oder dass es dieses Gefühl nur deshalb habe, weil es überempfindlich, undiszipliniert oder nicht positiv eingestellt sei.

Kompensationsmechanismen. Die fehlende Validierung emotionaler Erfahrungen führt zu einer wachsenden Diskrepanz zwischen den Erlebnissen des Kindes und dem, was durch die Umwelt bestätigt wird. Das Kind lernt darüber nicht, seine Gefühle zu benennen und seine emotionale Erregung zu regulieren. Aus diesem Grund versuchen die Patienten, ihre Emotionen weitestgehend zu vermeiden. Da dies jedoch langfristig nicht möglich ist, greifen sie auf schnell wirksame, langfristig zumeist jedoch sehr schädliche Verhaltensweisen zurück wie z. B. 
- selbstverletzendes Verhalten (SVV)

- Hochrisikoverhalten

- gestörtes Essverhalten

- Drogenkonsum

\section{Merke}

Suizidversuche stellen häufig die letzte Möglichkeit dar, sich der als unerträglich empfundenen emotionalen Zustände zu entledigen.

Primäre Ziele. Auf diese Annahmen aufbauend besteht das primäre Behandlungsziel der DBT darin, funktionale Strategien zur Emotionsregulation aufzubauen bzw. zu verbessern und gleichzeitig schweres Problemverhalten, wie z. B. suizidales oder schweres selbstschädigendes Verhalten, zu verringern. Können die Betroffenen ihr emotionales Erleben ohne den Einsatz dysfunktionaler Strategien regulieren, stehen im Fokus der Therapie

- soziale Isolation

- Arbeitslosigkeit

- eine komorbide PTBS

- andere komorbide psychische Störungen wie Depression, Angststörungen, Essstörungen, Zwangsstörungen

- andere Problembereiche

Endziele. Am Ende der Behandlung steht der Aufbau eines erfüllten Lebens auf der Grundlage von Selbstakzeptanz, Wertschätzung, Selbstfürsorge und achtsamem Selbstmitgefühl.

Gleichzeitig verfolgt die DBT als übergeordnetes Behandlungsziel, dialektische Verhaltensmuster herzustellen - ein Denken und Handeln, welches von Ausgewogenheit und Augenmaß bestimmt ist und von „ImMoment-Sein“. Die Auflösung dichotomer Betrachtungsweisen von sich und seiner Umgebung und damit ein tiefes inneres Annehmen von dem, was ist, stellt damit das attraktive Endziel der DBT dar.

Durchführung. Die ambulante Therapie ist für einen Zeitraum von 1-3 Jahren angelegt. In seltenen Fällen kann sie diesen Zeitraum aber auch überschreiten. Der Ablauf der Therapie ist klar strukturiert und lässt sich in eine Vorbereitungsphase und 3 sich daran unmittelbar anschließende Therapiephasen unterteilen.

\section{Vorbereitungsphase}

Analyse und Therapievertrag. Für die Vorbereitungsphase werden im ambulanten Kontext in der Regel zwischen 3 und maximal 10 Sitzungen benötigt. Sie umfasst die aktuellen Beschwerden, die Anamnese und Diagnostik. Darüber hinaus findet in der Vorbereitungsphase die Informationsvermittlung über das Krankheitsbild, die Darstellung des biosozialen Ätiologiemodells (s.o.), die Aufklärung über die Therapie im Allgemeinen und die DBT im Besonderen und die Ziel- und
Motivationsanalyse statt. Anhand von 1-2 detaillierten Verhaltens- und Bedingungsanalysen werden die entscheidenden Problembereiche eruiert, wie z. B. Suizidversuche, schweres SVV, starke Dissoziationen, fortwährende stationäre Aufenthalte und soziale Desintegration. Anschließend unterzeichnen der Patient und der Therapeut gemeinsam einen Therapievertrag, welcher die wichtigsten Therapieziele und die Einhaltung bestimmter Therapievereinbarungen zum Inhalt hat. Unter anderem verpflichtet sich der Patient, während der gesamten Therapiedauer keinen Suizidversuch zu unternehmen.

\section{Merke \\ Der Therapievertrag bedeutet eine tiefe innere Ver- pflichtung, die von beiden Seiten - Patient und The- rapeut - eingegangen wird. Die Unterschrift doku- mentiert diese Entscheidung.}

\section{Gravierende Komorbiditäten zuerst behandeln.} Kann ein erfolgreicher Verlauf der Therapie aufgrund gravierender komorbider Störungen nicht erwartet werden, sollten diese vorrangig behandelt werden. Dies gilt insbesondere für komorbide Störungen, die zu schweren funktionalen Einschränkungen bis hin zu lebensbedrohlichen Zuständen führen. Hierzu zählen z. B. psychotische Erkrankungen, schwerstes Untergewicht, Drogen-/Alkoholintoxikationen.

\section{TIPP FÜR DIE PRAXIS}

Erst nach der erfolgreichen Behandlung gravierender komorbider Störungen kann mit der DBT begonnen werden.

\section{Erste Therapiephase}

Einzeltherapie und Skillstraining. Die erste Therapiephase beginnt nach der Zustimmung zum Therapievertrag und dauert zwischen 4 Monaten und 2 Jahren, regelhaft ein Jahr. Sie hat zum Ziel, die in der Vorbereitungsphase definierten Problembereiche zu bearbeiten, wobei schwere dysfunktionale Verhaltensweisen innerhalb der ersten 3-4 Monate deutlich reduziert, wenn möglich sogar eingestellt werden sollten. Um dies zu ermöglichen, nehmen die Patienten parallel zur Einzeltherapie an einem gruppenbasierten Fertigkeitentraining teil, in dem Alternativen zu bislang dysfunktionalen Verhaltensweisen vermittelt werden.

Hierarchie der Problembereiche. Die Problembereiche sind hierarchisch nach Gefährlichkeit unterteilt (siehe Kasten „Therapiephasen und Hierarchie“): Wann immer ein höher geordneter Problembereich auftritt, z.B. schweres SVV, muss dieser unmittelbar in Form einer Verhaltensanalyse/Kettenanalyse sowie einer Lösungsanalyse behandelt werden. 
THERAPIEPHASEN UND HIERARCHIE DER JEWEILIGEN PROBLEMBEREICHE (ANGELEHNT AN [9])

\section{Vorbereitungsphase}

- Erheben der aktuellen Beschwerden, der Anamnese und der Diagnostik

- Informationsvermittlung über das Krankheitsbild

- Aufklärung über die Therapie im Allgemeinen und über die DBT im Besonderen

- Darstellung des biosozialen Ätiologiemodells

- Motivations- und Zielanalyse

- Zustimmung zu den Behandlungszielen, Behandlungsvertrag

Erste Therapiephase: Behandlung von schweren Problemen auf der Verhaltensebene

- Verringern von lebensbedrohlichem Verhalten/ Verbesserung der Überlebensfertigkeiten

- Suizidversuche

- fremdgefährdendes Verhalten (z. B. Totschlag)

- potenziell lebensbedrohliche unbehandelte medizinische Zustände

- Hochrisikoverhalten

- lebensbedrohliches SVV

- Verringern von Verhalten, das zu einem Therapieabbruch führt und Verbesserung von therapieaufrechterhaltendem Verhalten

- Burnout des Therapeuten (z. B. durch Bedrohung oder schwerwiegende Verunsicherung des Therapeuten)

- schwere Probleme im therapeutischen Setting (z. B. Nichterscheinen)

- Finanzierungsprobleme

- Verringern von Krisen generierendem Verhalten/Behandlung von sehr schwerwiegenden

Problemen

- schwerwiegende familiäre Probleme (z. B. Jugendamt)

- fatale Partnerschaften

- anhaltende Bedrohung von, oder sexuelle Kontakte mit Tätern

- ungeplante psychiatrische notfällige Aufnahmen

- rezidivierendes schweres SVV

- Verringern von Verhalten, das den Therapiefortschritt behindert und Verbesserung der Mitwirkung bei der Therapie

- Intoxikationen

- Dissoziationen

- chronisch anhaltende psychotische Symptome

- Major Depression

- schwere ADHS

- schwere PTBS
- Non-Compliance

- schwere Schlafprobleme

- schwere Ess- und Trinkstörungen

- schwere hormonelle Probleme (z. B. PMS)

- Verbesserung der Lebensqualität und Behandlung von sehr schwerwiegenden psychischen Störungen oder Problemen, die normale soziale Interaktionen und berufliche Tätigkeiten ver-

hindern

- Behandlung von weiteren schwerwiegenden Achse-I-Störungen (z. B. Essstörung, Abhängigkeitsstörung)

- Behandlung von Verhaltensmustern, die eine Verbesserung der sozialen Integration und Interaktion verhindern

- Rückführung auf den Arbeitsmarkt

- Zeitgleich: Aufbau und Verbesserung der Verhaltensfertigkeiten im Gruppensetting (Fertigkeitentraining)

- innere Achtsamkeit

- zwischenmenschliche Fertigkeiten

- bewusster Umgang mit Gefühlen

- Stresstoleranz

- Selbstwert

- Umgang mit Sucht (optional)

Zweite Therapiephase: Akzeptieren und Verändern von schwerem Leid auf der emotionalen Ebene

- weitere Reduktion posttraumatischer Symptome

- weitere Verbesserung der sozialen Integration und Interaktion

- weitere Unterstützung bei der Rückführung auf den Arbeitsmarkt

- weitere Behandlung der Achse-I-Störungen

- Behandlung Borderline-typischer emotionaler Probleme wie Einsamkeit, Angst vor Verlassenwerden, Selbsthass

Dritte Therapiephase: Probleme der Lebensführung akzeptieren und lösen

- Steigerung der Selbstachtung

- Steigerung der Wertschätzung

- Steigerung der Selbstfürsorge

- Steigerung des achtsamen Selbstmitgefühls

- Entwickeln und Umsetzen individueller Lebensziele

Wöchentliche Dokumentation. Die fortwährende Dokumentation der zuvor definierten Problembereiche findet über sog. Wochenprotokolle (Diary-card) statt (s. A Abb. 1). Zu Beginn einer jeden Sitzung besprechen Therapeut und Patient dieses Wochenprotokoll gemeinsam. Anhand der darin dokumentierten Verhal- 


\begin{tabular}{|c|c|c|c|c|c|c|c|c|c|}
\hline \multicolumn{10}{|c|}{ Wochenprotokoll-ambulant } \\
\hline \multicolumn{4}{|c|}{$\begin{array}{l}\text { Dialektisch-Behaviorale Therapie (DBT) } \\
\text { Wochenprotokoll }\end{array}$} & \multicolumn{5}{|c|}{ Name: ................. Frau K .......................... } & Start/Datum: ................................. \\
\hline \multirow[t]{3}{*}{ Datum } & \multirow{3}{*}{$\begin{array}{l}\text { Alkohol } \\
\text { (bitte } \\
\text { angeben) }\end{array}$} & \multirow{3}{*}{$\begin{array}{l}\text { nichtverordnete } \\
\text { Medikamente } \\
\text { (bitte angeben) }\end{array}$} & \multirow{3}{*}{$\begin{array}{l}\text { Medikamente } \\
\text { auf Rezept } \\
\text { (bitte angeben) }\end{array}$} & \multirow{3}{*}{$\begin{array}{l}\text { Drogen } \\
\text { (bitte } \\
\text { angeben) }\end{array}$} & \multirow{3}{*}{$\begin{array}{c}\text { suizidale } \\
\text { Vorstellungen } \\
(0-5)\end{array}$} & \multirow{3}{*}{$\begin{array}{l}\text { Not/ } \\
\text { Elend } \\
(0-5)\end{array}$} & \multicolumn{2}{|c|}{ Selbstschädigung } & \multirow{3}{*}{$\begin{array}{l}\text { angewandte } \\
\text { Methode } \\
\text { (Skills: } 0-7 \text { ) }\end{array}$} \\
\hline & & & & & & & & & \\
\hline & & & & & & & $\begin{array}{c}\text { Drang } \\
(0-5)\end{array}$ & $\begin{array}{l}\text { Handlung } \\
\text { (ja/nein) }\end{array}$ & \\
\hline Mo & & keine & & keine & 1 & 2 & 2 & nein & 6 \\
\hline $\mathrm{Di}$ & & keine & & keine & 2 & 3 & 2 & nein & 6 \\
\hline $\mathrm{Mi}$ & 1 Glas Wein & keine & & keine & 2 & 3 & 3 & nein & 6 \\
\hline Do & & keine & & keine & 1 & 1 & 1 & nein & 7 \\
\hline $\mathrm{Fr}$ & & keine & & keine & 2 & 3 & 3 & nein & 6 \\
\hline Sa & 1 Glas Wein & keine & & 1 Joint & 2 & 3 & 3 & nein & 6 \\
\hline So & & keine & & keine & 3 & 5 & 5 & ja & 2 \\
\hline \multicolumn{2}{|c|}{ Suizidale Ideen } & \multicolumn{2}{|c|}{ Selbstverletzungen } & \multicolumn{3}{|c|}{ Not/Elend bzw. Freude } & \multicolumn{2}{|l|}{ Skills } & \\
\hline \multicolumn{2}{|c|}{$0=$ keine } & \multicolumn{2}{|c|}{$0=$ keine } & \multirow{2}{*}{\multicolumn{3}{|c|}{$\begin{array}{l}0=\operatorname{kein}(e) \\
1=\operatorname{kaum}\end{array}$}} & \multicolumn{3}{|c|}{$0=$ nicht daran gedacht oder angewendet } \\
\hline \multicolumn{2}{|c|}{$1=$ kaum } & \multicolumn{2}{|l|}{$1=$ kaum } & & & & \multicolumn{3}{|c|}{1 = daran gedacht, nicht angewendet, wollte nicht } \\
\hline \multicolumn{2}{|c|}{2 = mäßig } & \multicolumn{2}{|c|}{2 = mäßig } & \multicolumn{3}{|c|}{$2=$ etwas } & \multicolumn{3}{|c|}{$2=$ daran gedacht, nicht angewendet, hätte gewollt } \\
\hline \multicolumn{2}{|c|}{$3=$ drängend } & \multicolumn{2}{|c|}{$3=$ starker Drang } & \multicolumn{3}{|c|}{$3=$ mittelmäßig } & \multicolumn{3}{|c|}{$3=$ habe es versucht, konnte sie aber nicht anwenden } \\
\hline \multicolumn{2}{|c|}{$4=$ sehr drängend } & \multicolumn{2}{|c|}{4 = sehr starker Drang } & \multicolumn{3}{|c|}{$4=$ groß } & $4=$ vers & sucht, konnt & halfen nicht \\
\hline $\begin{array}{r}5=\text { das } D \\
\text { komp } \\
\text { Ideen }\end{array}$ & $\begin{array}{l}\text { enken ist } \\
\text { lett auf suiz. } \\
\text { eingeengt }\end{array}$ & $5=$ nicht kor & trollierbar & $5=s$ & ehr groß & & $\begin{array}{l}5=\text { aut } \\
6=\text { vers } \\
7=\text { aut }\end{array}$ & $\begin{array}{l}\text { tomatisch an } \\
\text { sucht, konnt } \\
\text { tomatisch an }\end{array}$ & $\begin{array}{l}\text { n nicht } \\
\text { halfen } \\
\text { fen }\end{array}$ \\
\hline
\end{tabular}

Abb. 1 Wochenprotokoll anhand eines Fallbeispiels.

\section{BEISPIELDIALOG ZUM WOCHENPROTOKOLL}

Therapeut: Guten Tag Frau K. Schön, dass Sie da sind.

Patientin: Guten Tag.

$\mathrm{T}$ : Wie geht es Ihnen?

$P$ : Es geht so.

$\mathrm{T}$ : Was heißt das genau?

P: Naja, es gab schon bessere Tage.

T: Das hört sich danach an, dass es eher nicht so gut geht. Können Sie mal versuchen, Ihre aktuelle Verfassung in einer Skala zwischen - 5 und + 5 einzustufen? -5 bedeutet hierbei, dass es Ihnen ausgesprochen schlecht geht, 0 bedeutet neutral und +5 bedeutet, dass es ihnen nicht besser gehen könnte.

P: $\mathrm{Hm}$...-3:

T. Ok. Sind Sie trotzdem arbeitsfähig?

P: Geht schon.

T: Alles klar. Sollten Sie Unterstützung benötigen, dann geben Sie einfach Bescheid.

Kurze Pause

T: Haben Sie Ihr Wochenprotokoll dabei?
Frau K. reicht dem Therapeuten das ausgefüllte Wochenprotokoll.

T: Vielen Dank. Dann lassen Sie uns mal schauen. Seitdem wir uns vergangene Woche Mittwoch gesehen haben, haben Sie 2-mal Alkohol konsumiert. Aber wie ich sehe, jeweils nur ein Glas Wein. Am Samstag haben Sie zusätzlich einen Joint geraucht. Selbstverletzungen gab es letzte Woche keine, allerdings dann eine am Sonntag. Ach, das ist aber schade.

Kurze Pause

T: Haben Sie die Verhaltensanalyse dabei?

$P$ : Ja. Muss ich ja.

$\mathrm{T}$ : Das stimmt. Ich schau mal noch die anderen Spalten des Wochenprotokolls an. Die Suizidalität hatten Sie ja ganz gut im Griff; am Sonntag hatten Sie mal eine 3 eingetragen, sonst nur eine 1 oder 2. Not und Elend war so im mittleren Bereich, aber auch hier am Sonntag mit einer 5 deutlich erhöht. Scheint ein schwieriger Tag für Sie gewesen zu sein, der Sonntag, oder? P: Ja, das kann man so sagen. Irgendwie ging an dem Tag alles schief. 
Der Therapeut erstellt nachfolgend die Agenda für die Therapiestunde.

T: Okay. Dann müssen wir uns das mal mithilfe ihrer Verhaltensanalyse genauer anschauen. Gibt es denn sonst noch etwas, was wir dringend besprechen müssen?

P: Ich stehe im Moment bei der Arbeit ganz schön unter Druck. Ich glaube, dass es mir deswegen auch am Sonntag so schlecht ging, weil am Montag ja wieder Arbeitstag war.

$\mathrm{T}$ : Dann sollten wir uns darum ebenfalls noch kümmern. Ich nehme aber mal an, dass wir uns im Rahmen der Lösungsanalyse ohnehin um ihre Arbeitssituation kümmern werden, wenn diese ein Auslöser für ihr SVV am Sonntag war.

P: Okay.

$\mathrm{T}$ : Wie war das Fertigkeitentraining letzte Woche Donnerstag? Waren Sie dort?

P: Ja. War ganz okay.

$\mathrm{T}$ : Was ist aktuell Thema?

P: Stresstoleranz.

T: Okay, das passt ja ganz gut. Vielleicht haben Sie dort ja noch ein paar Fertigkeiten vermittelt bekommen, um SVV in Zukunft zu verhindern. Dann schlage ich vor, dass wir nun mit der Besprechung Ihrer Verhaltensanalyse beginnen, dann erarbeiten wir uns die Lösungsanalyse und am Schluss schauen wir mal, dass wir noch $5 \mathrm{Mi}$ nuten Zeit haben, um über Ihre Arbeitssituation zu sprechen.

tensweisen strukturiert der Therapeut dann die Therapiestunde.

Kontingenzmanagement. Die Problembereiche zu hierarchisieren, hat maßgebliche Implikationen für den Therapieverlauf: Solange beispielsweise wöchentlich schweres SVV vorliegt, muss jede Woche eine Verhaltensanalyse/Kettenanalyse und Lösungsanalyse in der Einzeltherapie besprochen werden. Alle anderen zusätzlich vorhandenen Problemfelder können unterdessen nicht oder nur sehr kurz thematisiert werdenvorausgesetzt, es tritt kein hierarchisch höhergeordnetes Problemverhalten auf. Dies frustriert den Patienten zunehmend, aber auch den Therapeuten und das Konsultationsteam (s.u.). Folge dieses Kontingenzmanagements ist optimalerweise eine Erhöhung des Veränderungsdrucks, der notwendig sein kann, um die Angst vor Veränderungen zu überwinden. Damit orientiert sich die DBT im Unterschied zu den meisten anderen Manualen zur Behandlung spezifischer Störungsbilder nicht an einer festgelegten Reihenfolge von Sitzungen und Inhalten, sondern dynamisch an den von den Patienten gezeigten Verhaltensmustern.
TIPP FÜR DIE PRAXIS

Die Fortsetzung der DBT wird vom erfolgreichen Verlauf der Behandlung abhängig gemacht. Ohne eine absehbare sowie evaluierbare, zielkonforme Veränderung muss in diesem Fall die Effektivität der DBT infrage gestellt werden. Gelegentlich kann eine Therapiepause ein probates Mittel zur Überprüfung der Therapiemotivation/des Therapiecommitments sein. Diese sollte wann immer möglich einem endgültigen Therapieende vorgeschaltet werden.

\section{Zweite Therapiephase}

Die zweite Therapiephase beginnt, wenn schweres dysfunktionales Problemverhalten weitestgehend eingestellt werden konnte. Sie erstreckt sich in der Regel über 1 - 2 Jahre. Diese Therapiephase hat zum Ziel, weitere emotionale Probleme zu bearbeiten sowie bislang nicht zugelassene Aspekte des Lebens zu integrieren. Hierzu zählt die (weitere) Verbesserung der beruflichen wie auch partnerschaftlichen Situation sowie die Behandlung von in der ersten Therapiephase noch nicht behandelten psychischen Störungen (z. B. Essstörungen, Drogenmissbrauch, soziale Phobie). Bei Bestehen einer komorbiden PTBS ist ein wesentliches Ziel dieser zweiten Therapiephase, die möglicherweise bereits begonnene Traumabehandlung fortzuführen. Für das spezifische Vorgehen zur Behandlung einer PTBS im Rahmen einer DBT-Behandlung wird auf die einschlägige Literatur verwiesen („DBT-PTSD“; [5]).

\section{Merke \\ Es hat sich mittlerweile durchgesetzt, mit der PTBS- Behandlung möglichst frühzeitig zu beginnen.}

Störungsspezifische Protokolle. Haben sich bestimmte funktionale Verhaltensweisen zuungunsten bislang dysfunktionaler Verhaltensweisen etabliert, kann auf deren Dokumentation im Rahmen des Wochenprotokolls zunehmend verzichtet werden. Sofern dies noch nicht geschehen ist, kann es stattdessen nun hilfreich sein, noch vorhandene Problembereiche mit hierfür entwickelten störungsspezifischen Protokollen zu erfassen, beispielsweise Essstörungen mit einem Essprotokoll.

Therapiephasen an Fortschritte anpassen. Kommt es wieder zu länger währenden schwerwiegenden Problemen auf der Verhaltensebene, wie sie für die erste Therapiephase kennzeichnend waren, wird die zweite Therapiephase ausgesetzt und zur Bearbeitung analog der ersten Therapiephase zurückgekehrt. Auch das Wochenprotokoll wird dann zumeist wieder aufgegriffen. 


\section{Dritte Therapiephase}

Ziel. Die dritte Therapiephase soll die Selbstachtung, die Wertschätzung gegenüber anderen und sich selbst, die Selbstfürsorge sowie das achtsame Selbstmitgefühl steigern. Marsha Linehan formulierte: „In diesem Stadium der Therapie hat die DBT große Ähnlichkeit mit psychodynamischen und klientenzentrierten Ansätzen" ([1], S. 132). Besonders empfehlenswert sind Therapieprogramme von Potreck-Rose und Jacob, Christopher Germer und Paul Gilbert [6-8]. Fokus der beiden zuletzt genannten Therapieprogramme ist Selbstmitgefühl, wobei Chris Germer die enge Verbindung zur Achtsamkeit betont, Paul Gilbert hingegen vermehrt die therapeutische Umsetzung fokussiert.

Dauer. Da die dritte Therapiephase zumeist innerhalb der zweiten Therapiephase - und in einem geringeren Umfang durchaus auch schon während der ersten Therapiephase - beginnt, handelt es sich hierbei um keine abgeschlossene Therapiephase. Die Dauer der dritten Therapiephase kann daher sehr unterschiedlich sein.

\section{Therapieelemente}

\author{
DIE 5 BAUSTEINE DER DBT \\ - Einzeltherapie \\ - Fertigkeitentraining in der Gruppe \\ - Telefonkontakte \\ - Konsultationsteam \\ - Supervision
}

\section{Einzeltherapie}

Die in der Regel einmal wöchentlich stattfindende Einzeltherapie ist zuständig für die individuelle Ziel- und Motivationsarbeit sowie die Durchführung von Problem- und Lösungsanalysen. Gleichzeitig werden in der Einzeltherapie fortwährend die Emotionen des Patienten fokussiert, wobei zu Beginn der Therapie v.a. die Kontrolle, später das Zulassen und Akzeptieren der Emotionen vorrangig ist.

\section{Merke}

Die DBT ist in erster Linie eine emotionsfokussierte und weniger eine kognitive Therapie.

\section{Fertigkeitentraining in der Gruppe}

Parallel zur Einzeltherapie muss ein Fertigkeitentraining besucht werden. Dieses findet wöchentlich statt, dauert jeweils 1,5-2 Zeitstunden und wird in einer Gruppe von maximal 7-8 Patienten durchgeführt. Zwei Trainer leiten das Training. Ziel des Fertigkeitentrainings ist die Vermittlung von spezifischen Fertigkei-
BEISPIELDIALOG ZUM EMOTIONSFOKUSSIERTEN VORGEHEN

Therapeut: Schauen Sie mal genauer hin, welche Emotion gerade hinter der aktuellen Spannung stehen könnte.

Patientin: Ich habe keine Ahnung. Ich spüre nur Spannung. Und die ist unerträglich.

T: Okay. Wenn die Spannung zu hoch ist, dann ist es in der Tat unmöglich, die dahinter liegende Emotion wahrzunehmen. Wie hoch ist denn aktuell die Spannung auf einer Skala zwischen 0 und $100 ?$

P: 80 bis 90 .

T: Das ist hoch. Können Sie Fertigkeiten einsetzen, um die Spannung jetzt zu reduzieren? Die Patientin wendet mithilfe des Therapeuten Stresstoleranzfertigkeiten an, allen voran Ablenkung (z. B. Beschreibung eines Bildes).

$\mathrm{T}$ : Wie hoch ist die Spannung nun?

P: 60

T: Okay. Können Sie nun die Emotion wahrnehmen?

P: Ich weiß nicht so recht. Könnte Ärger sein.

$\mathrm{T}$ : Woran merken Sie das?

Nachfolgend erfragt der Therapeut einzelne

Bestandteile des emotionalen Netzwerks (Wahrnehmung, Körper, Gefühl, Handlungsimpuls, Gestik/Mimik), mit dem Ziel, die zugrunde liegende Emotion besser zu erfassen sowie sie zu validieren und damit das Ausmaß der Emotion zu reduzieren.

P: Dass ich meine Fäuste balle. Und dass ich in meinem Magen so einen Druck verspüre, wie eine Kugel, die sich dreht.

T: Auf wen oder was sind Sie denn wütend?

P: Auf meinen Partner. Weil er mir vorhin für heute Abend abgesagt hat.

$\mathrm{T}$ : Was würden Sie jetzt am liebsten machen? $\mathrm{P}$ : Meinen Partner anrufen und ihm sagen, wie blöd ich das von ihm finde.

T: Ja, das hört sich sehr nach Wut und Ärger an. Was passiert, wenn Sie Ihre Wut so beschreiben, wie Sie das gerade gemacht haben? $\mathrm{P}: \mathrm{Hm}$... Wird ein bisschen weniger.

\section{DEFINITION FERTIGKEITEN}

Unter Fertigkeiten („Skills“) werden kognitive, emotionale und handlungsbezogene Reaktionen verstanden, die sowohl kurz- als auch langfristig zu einem Maximum an positiven und einem Minimum an negativen Ergebnissen führen. 
ten, welche für ein therapeutisches Fortkommen als unverzichtbar angesehen werden.

Die Fertigkeiten sind nach Bohus und Wolf in 5, optional 6 Module unterteilt [10]:

- Fertigkeiten zur Steigerung der inneren Achtsamkeit

- zwischenmenschliche Fertigkeiten

- Fertigkeiten zum bewussten Umgang mit Gefühlen

- Fertigkeiten zur Stresstoleranz

- Fertigkeiten zur Steigerung des Selbstwertes

- optional Fertigkeiten zum Umgang mit Sucht

Zu jedem Modul existieren spezifische Arbeits- und Übungsblätter. Die Länge eines Moduls beträgt 4-9 Wochen. Jedes Modul wird in der Regel 2-mal vermittelt, womit die Gesamtdauer des Fertigkeitentrainings ca. $1 \frac{1}{2}$ Jahre beträgt.

\section{Telefonkontakte}

Telefonkontakte dienen dem Transfer des bereits Gelernten in den Alltag des Patienten. Der Patient kann den Therapeuten im Rahmen von vorher klar definierten Zeiträumen in akuten Krisen anrufen (z. B. zwischen 9 und 19 Uhr, außer am Wochenende; Rückrufgarantie innerhalb von 24 Stunden, außer am Wochenende). Innerhalb eines solchen Telefonats vermittelt der Therapeut effektive Fertigkeiten, um mit der aktuellen Situation umgehen zu können. Das Gespräch sollte nicht länger als 5 bis maximal 10 Minuten dauern.

\section{Konsultationsteam}

Das ambulante Konsultationsteam besteht aus 3-6 ambulanten Therapeuten und Fertigkeitentrainern, die sich wöchentlich für ca. eine Zeitstunde treffen. Aufgabe des Konsultationsteams ist zweierlei:

- Zum einen hat das Team dafür Sorge zu tragen, dass der Patient die bestmögliche DBT erhält.

- Zum anderen ist die Aufgabe des Teams, die Behandlung gemeinsam durchzuführen und damit den Einzeltherapeuten zu entlasten.

Sämtliche therapeutischen Schritte werden im Team beschlossen und sind anschließend von dem zuständigen Einzeltherapeuten umzusetzen. Im Unterschied zu den meisten anderen Therapien wird die Behandlung demnach nicht von einer Einzelperson, sondern von einem Therapeutenteam durchgeführt.

TIPP FÜR DIE PRAXIS

Es hat sich bewährt, dass sich die im Konsultationsteam behandelten Patienten nach der Unterzeichnung des Therapievertrags einmalig dem Team unter Angabe ihrer Therapieziele vorstellen.

\section{FALLBEISPIEL}

Eine Borderline-Patientin verletzte sich mindestens einmal in der Woche so schwer, dass jeweils eine chirurgische Behandlung notwendig war. Die behandelnde Einzeltherapeutin musste jede Woche im Konsultationsteam von diesem Verhalten berichten, da es sich hierbei um zielrelevantes dysfunktionales Verhalten handelte. Im Rahmen der Besprechung wurde deutlich, wie sehr die fortwährende Besprechung von Verhaltensanalysen die Einzeltherapeutin belastete. Diese bekam daraufhin den Auftrag, die Patientin hierüber zu informieren, mit der Bitte, einen Zeitraum zu definieren, in welchem kein schweres SVV mehr auftreten durfte. Gleichzeitig sollte die Einzeltherapeutin erfragen, was die Patientin zur Umsetzung dieser Auflage benötigt.

Beim nächsten Konsultationsteam berichtete die Einzeltherapeutin dann von dem Ergebnis dieser Mitteilung.

\section{Supervision}

Individuelle Prozesse und Schwierigkeiten, die im Rahmen des Konsultationsteams nicht aufgefangen und bearbeitet werden können, werden in der zusätzlichen Supervision besprochen (z. B. die Schwierigkeit, unangenehme Konsequenzen durchzusetzen; zwischenmenschliche Schwierigkeiten zwischen einzelnen Mitgliedern des Konsultationsteams).

\section{Die therapeutische Grundhaltung}

Erster Grundpfeiler der DBT. Stellt die beschriebene Struktur den Rahmen der DBT dar, bildet die therapeutische Grundhaltung neben den zum Einsatz kommenden therapeutischen Techniken und Strategien (siehe nächstes Kapitel) einen der bereits erwähnten Grundpfeiler der DBT.

Grundannahmen als Schutz vor Vorurteilen. Aufgrund der weitverbreiteten Vorurteile gegenüber Borderline-Patienten und deren Behandlung wurde es notwendig, Grundannahmen explizit zu formulieren, welche die Basis der therapeutischen Grundhaltung definieren. Die erwähnten Vorurteile sind dem Umstand geschuldet, dass die Arbeit mit Borderline-Patienten zumeist als sehr anstrengend erlebt wird. Insbesondere die hohe Emotionalität und die stellenweise sehr belastenden dysfunktionalen Verhaltensweisen (schweres SVV, Suizidandrohungen, Suizidversuche) stellen hohe Herausforderungen an die therapeutische Kompetenz dar. Eine Abbruchrate von bis zu 67\% im Rahmen nicht standardisierter ambulanter Therapien belegt dies eindrucksvoll (z. B. [11]). Den Patienten wird häufig vorgeworfen, sich „manipulativ“ zu verhalten, sich „nicht 
ausreichend anzustrengen“, akute Suizidalität unmittelbar vor dem Urlaub des Therapeuten bewusst als „Erpressungsversuch“ einzusetzen. Da diese häufig zu beobachtenden Vorurteile ein zufriedenstellendes Behandlungsergebnis ernsthaft gefährden können, dienen die nachfolgend aufgeführten 8 Grundannahmen sowohl dem Schutz des Patienten wie auch des Therapeuten.

\section{Merke}

Den Grundannahmen liegt eine dialektische Sichtweise zugrunde: der Therapeut muss die Patienten so akzeptieren wie sie sind; gleichzeitig muss er die Patienten dabei unterstützen, sich in Übereinstimmung mit ihren Zielen zu verändern.

THERAPEUTISCHE GRUNDANNAHMEN

1. Borderline-Patienten geben sich wirklich Mühe. Das heißt, sie versuchen, das Beste aus ihren gegenwärtigen Situationen zu machen.

2. Borderline-Patienten wollen sich verändern.

3. Borderline-Patienten müssen sich stärker anstrengen und härter arbeiten, um sich zu verändern.

4. Borderline-Patienten haben ihre Schwierigkeiten nicht selbst verursacht, müssen sie aber selbst lösen.

5. Das Leben suizidaler Borderline-Patienten ist so wie es gegenwärtig ist unerträglich.

6. Borderline-Patienten müssen neues Verhalten in allen relevanten Lebensbereichen erlernen.

7. Patienten können in der Therapie nicht versagen.

8. Therapeuten, die mit Borderline-Patienten arbeiten, brauchen Unterstützung.

Grundannahme 1. Hier liegt die Annahme zugrunde, dass Patienten sich häufig in emotionalen Zuständen befinden, wie sie andere nur in lebensbedrohlichen Situationen erleben (s. biosoziales Ätiologiemodell).

Grundannahme 2. Sie unterstellt, dass der Patient aufgrund der Unerträglichkeit der Lebenssituation notgedrungen bereit ist, sich zu verändern - auch wenn manchmal ein gegenteiliger Eindruck vorherrschen mag.

Grundannahme 3. Diese Annahme betont zum einen die bereits geleisteten Anstrengungen des Patienten. Sie macht aber auch deutlich, dass Veränderungen anfänglich noch mehr Anstrengung erfordern. Denn sie gefährden das mühevoll, wenn auch sehr fragile Gleichgewicht, welches der Patient bislang aufrechterhalten hat.
Grundannahme 4. Diese validiert die Wut und die Ohnmacht, die angesichts der unausweichlichen Anstrengung, die für eine Veränderung notwendig ist, entstehen mag; sie macht aber auch die Eigenverantwortung des Patienten für die Umsetzung dieser Veränderung deutlich.

Grundannahme 5. Sie greift die Verzweiflung des Patienten angesichts der erlebten emotionalen Not auf.

Grundannahme 6. Dieser 6. Grundannahme entsprechend gilt es, fortwährend unterstützend und motivierend auf den Patienten einzuwirken, den einmal eingeschlagenen Weg weiterzuverfolgen, die Veränderungen auf alle Lebensbereiche auszudehnen.

Grundannahme 7. Diese macht deutlich, dass die Schuld für ein Versagen der Therapie niemals beim Patienten zu suchen ist, sondern die DBT nicht in jedem Fall über ausreichende Strategien verfügt, um den Patienten zu neuem Verhalten zu verhelfen. Neben der DBT gibt es weitere evaluierte Therapieverfahren zur Behandlung einer Borderline-Störung, die unter den gegebenen Umständen die möglicherweise passenderen Techniken anbieten:

- Schema-Fokussierte Therapie, SFT [12]

- Mentalisierungsbasierte Therapie, MBT [13]

- Übertragungs-fokussierte Therapie, TFP [14]

- Therapeutisches Vorgehen entsprechend den Richtlinien der American Psychiatric Association (APA) [15]

Grundannahme 8. Sie schützt den Therapeuten vor einem frühzeitigen Ausbrennen und sorgt damit dafür, dass der Patient immer die bestmögliche DBT erhält (s. Konsultationsteam).

\section{Therapeutische Strategien}

Merke

In der DBT kommen sowohl akzeptanzbasierte Strategien wie auch veränderungsorientierte Strategien gleichberechtigt zum Einsatz. Die aus der Gegensätzlichkeit dieser beiden Strategien entstehende Spannung wird für den therapeutischen Prozess gewinnbringend genutzt.

Zweiter Grundpfeiler der Therapie. Die nachfolgend dargestellten Techniken/Strategien stellen den zweiten Pfeiler der DBT dar. Kennzeichnend für die DBT ist der Einsatz von 2 sich widersprechenden therapeutischen Strategien:

- veränderungsorientierte Strategien

- akzeptanzbasierte Strategien

So haben beispielsweise Borderline-Patienten zwar ihr Leid häufig nicht selbst verschuldet (Akzeptanz), aber 
sie sind die einzigen, die dieses Leid beenden können (Veränderung). Die durch die Unvereinbarkeit entstehende Spannung sorgt für den nötigen Druck, aber auch für die notwendige Energie, um eine Änderung herbeizuführen. Der Patient fängt automatisch an, den Druck reduzieren zu wollen, indem er mögliche Lösungen dieses Dilemmas reflektiert.

Akzeptanz und Veränderung im Wechsel. Erfahrene DBT-Therapeuten zeichnen sich durch einen raschen, spielerisch wirkenden Wechsel zwischen diesen beiden Polen aus, sodass die gegebenen Dichotomisierungstendenzen von Borderline-Patienten aufgefangen werden und die Patienten lernen, ihre Probleme dialektisch zu betrachten. Marsha Linehan beschreibt diesen Prozess als einen gemeinsamen Tanz zwischen dem Patienten und dem Therapeuten auf dem Boden einer energiegeladenen Tanzfläche.

Spannung nicht zu früh beenden. Unerfahrene Therapeuten zeichnen sich hingegen häufig dadurch aus, dass sie die bei Borderline-Patienten zugegebenermaßen sehr starke Spannung nicht aushalten und vorzeitig für eine Beendigung der Spannung sorgen, z. B. indem sie frühzeitig mit Veränderungsvorschlägen aufwarten oder an sich sinnvolle Anforderungen zu früh reduzieren (z. B. nicht konsequent genug darauf drängen, schweres SVV aufzugeben).

Akzeptanzbasierte Strategien. Den beiden therapeutischen Strategien werden bestimmte Techniken zugeordnet. Zu den akzeptanzbasierten Strategien zählen:

- Techniken der Problemerhebung

- Validierung

- manche Kommunikationstechniken

Veränderungsstrategien. Diese Techniken werden ausbalanciert durch Veränderungsstrategien wie:

- Problemlösung

- Kontingenzmanagement

- Exposition

- kognitive Umstrukturierung

- der überwiegende Teil der Kommunikationsstrategien

- Vermittlung von Fertigkeiten

Dialektische Strategien. Zusätzliche dialektische Strategien regeln den angemessenen Einsatz dieser beiden gegensätzlichen Strategieblöcke.

Strukturelle Strategien Strukturelle Strategien stellen für das gesamte therapeutische Vorgehen den funktionalen Rahmen.

\footnotetext{
Merke

Validierungsstrategien stellen die Grundlage einer jeden Veränderung dar!
}

BEISPIELDIALOG ZUM EINSATZ VON VALIDIERUNGSSTRATEGIEN VS. EINSATZ VON VERÄNDERUNGSSTRATEGIEN:

Patientin: Das Leben ist unerträglich. Ich weiß nicht mehr weiter. Ich kann einfach nicht mehr! Nachfolgend validiert der Therapeut die Patientin.

Therapeut: Oh weh! Das hört sich danach an, dass es Ihnen gerade richtig schlecht geht.

P: Das ist noch gar kein Ausdruck, wie es mir tatsächlich gerade geht.

T. Verzweiflung? Hoffnungslosigkeit?

P: Und wie!

T: Was ist denn vorgefallen, dass es Ihnen so schlecht geht?

P: Mein Freund hat mich verlassen.

T: Ach du meine Güte! Das ist ja entsetzlich!

P: Ja! Am Telefon hat er mir mitgeteilt, dass er sich trennen möchte.

T: Am Telefon? Was ist das denn für ein Mist!

Patientin weint.

T: Das tut mir so leid, Frau S. Was müssen Sie gerade Schlimmes durchmachen!

$\mathrm{P}$ : Ich werde doch immer verlassen! Ich bin einfach nicht zum Aushalten!

Nachfolgend setzt der Therapeut Veränderungsstrategien ein.

T: Haaalt! Ich sehe Ihren Schmerz und finde es auch völlig nachvollziehbar, dass Sie zutiefst verletzt, gekränkt und verunsichert sind. Aber hilft es ihnen weiter, wenn Sie sich jetzt zusätzlich beschimpfen und fertig machen? Geht es Ihnen damit besser?

$P$ : Nee. Ich weiß schon. Aber es tut so weh! T: Absolut! Aber vielleicht wäre es daher besser, wenn Sie überlegen, was Sie machen können, damit es IHNEN wieder bessergeht?

Validierungsstrategie. Die Bereitschaft zur Veränderung entsteht bei dem Patienten erst, wenn er sich ausreichend von seinem Gegenüber verstanden und gesehen fühlt. Aus diesem Grund geht in den meisten Fällen dem Einsatz einer Veränderungsstrategie der Einsatz einer Validierungsstrategie voraus. Diese Regel findet nur in extremen Anspannungszuständen des Patienten keine Anwendung.

\section{Die therapeutische Beziehungs- gestaltung}

Die Beziehungsgestaltung in der DBT ist ungewöhnlich intensiv und verbindlich. Sie ist herzlich, authentisch und freundlich. Sie ist die Basis einer jeden Intervention, das Sprungbett zur Veränderung. Umso angstbe- 
BEISPIELDIALOG (AM TELEFON) ZUR REAKTION

AUF FUNKTIONALES UND DYSFUNKTIONALES

VERHALTEN

Verstärken von funktionalem Verhalten über die Beziehung, „Bestrafung“ von dysfunktionalem Verhalten über Rückzug

Patientin: Ich habe einen solch hohen Schneidedruck, ich kann einfach nicht mehr aufhören, mich selbst zu verletzen.

Therapeut: Verstehe ich Sie richtig, dass Sie sich wieder Rasierklingen besorgt haben?

P: Ja.

Nachfolgend ist der Therapeut harsch, konfrontativ, kurz angebunden.

T: Mein Vorschlag ist, dass Sie die Rasierklingen jetzt entfernen.

P: Ich kann das nicht.

T: Was können Sie nicht? Sie können die Hand nicht mehr bewegen, die die Rasierklingen beseitigen soll?

P: Nein, das schon. Ich kann Sie aber nicht wegschmeißen.

T: Das heißt, Sie wollen sich weiter schneiden?

$P$ : Nein, das auch nicht.

T: Frau K. Ich glaube es wäre sinnvoll, wenn Sie sich jetzt entscheiden. Ich respektiere es, sollten Sie sich dafür entscheiden, dass Sie die Rasierklingen behalten. Aber ich garantiere Ihnen in diesem Fall, dass der Schneiddruck nicht weniger wird. Und dann können wir damit eigentlich auch das Telefonat beenden, oder? Weil dann entscheiden Sie sich ja für die alte Lösung.

P: Oh Gott. Ja! Ich schmeiß sie weg!

Nachfolgend wird der Therapeut weich, zugewandt, fürsorglich. Entsprechend verändert sich auch die Tonlage des Therapeuten.

T: Ach, Frau K.! Ich weiß, wie schwierig das ist. Zumindest kann ich mir das vorstellen. Wissen Sie was: Ich bleibe noch so lange bei Ihnen, bis die Rasierklingen weg sind und Sie eine alternative Lösung gefunden haben. Und ich bin absolut sicher, dass Sie eine andere Lösung finden werden!

setzter die Veränderung, desto wichtiger ist die Beziehung. Die Beziehung ist ein gemeinsamer Teppich, auf dem der Therapeut und der Patient sitzen, an dem der Therapeut und der Patient permanent arbeiten.

Authentische Reaktion des Therapeuten. Marsha Linehan beschreibt eine gut funktionierende therapeutische Beziehung wie einen gemeinsamen Tanz („Playing Jazz“). Sie ist Leichtigkeit und Spannung zugleich; sie ist Akzeptanz und Veränderung in einem. Funktionales Verhalten verstärkt der Therapeut fortwährend

\section{BEISPIELDIALOG ZU GRENZEN}

Therapeut: Frau K. Ich muss Ihnen mitteilen, dass Sie mich in den letzten 2 Wochen meinem Empfinden nach zu häufig angerufen haben.

Patientin: Aber Sie haben doch gesagt, dass ich Sie anrufen soll, wenn ich nicht mehr weiterweiß. T: Sie haben völlig recht. Trotzdem gibt es für mich eine Grenze, wie viele Telefonate ich meistern kann. Ich habe ja auch noch ein Privatleben, 2 Kinder und eine Frau. Da kann ich nicht jeden Tag mehrmals mit Ihnen telefonieren. Das geht mal für eine Woche, aber nicht länger. Ist das nachvollziehbar für Sie?

P: Ja, aber was soll ich denn nun machen? T: Wenn Sie möchten, können wir uns darüber unterhalten, was Sie benötigen, dass Sie nicht mehr so häufig anrufen müssen.

über die Beziehung, ist zugewandt und herzlich. Auf dysfunktionales Verhalten hingegen reagiert der Therapeut eher mit Rückzug, kurzen, knappen Sätzen und harsches Verhalten. Demnach ist der Therapeut unabhängig von dem Verhalten des Patienten nicht gleichbleibend freundlich, sondern er reagiert authentisch; identisch zu der Reaktion gegenüber einem guten Freund oder einer guten Freundin.

\section{Merke}

In der DBT wird dysfunktionales Verhalten niemals über die Beziehung verstärkt, funktionales Verhalten hingegen immer.

Balance wie auf einer Wippe. In der DBT gibt es für das Vorgehen im Rahmen der Beziehungsgestaltung die Metapher der Kinderwippe („Teeter-Totter“). Entsprechend dieser Metapher befinden sich sowohl der Therapeut wie auch der Patient stehend auf dieser Wippe, jeder auf seiner Seite. Bewegt sich der Patient weg von dem Therapeuten, geht dieser ebenfalls nach hinten, um die Balance wiederherzustellen. Bewegt sich der Patient nach vorne, geht der Therapeut ebenfalls nach vorne. Droht der Patient über die Mittelachse auf die Seite des Therapeuten zu kommen (z. B. möchte der Patient so viel Nähe/Kontakt, dass die Grenzen des Therapeuten überschritten werden), dann macht der Therapeut dies unmittelbar klar und bittet den Patienten, wieder auf Abstand zu gehen.

\section{Effektivität und Modifikationen}

\section{Effektivität der DBT}

Eingang in Leitlinien. Die Wirksamkeit der DBT zur Behandlung einer BPS konnte in zahlreichen randomisiert-kontrollierten klinischen Studien (RCTs) belegt werden (Übersicht bei [16]). Stoffers und Mitarbeiter 
schlussfolgern im Rahmen ihrer Metaanalyse, dass DBT zur Behandlung der BPS das bis dato einzige Verfahren ist, welches auf mehrere RCTs zurückgreifen kann [16]. In den S2-Leitlinien für Persönlichkeitsstörungen deutscher Fachgesellschaften (DGPPN) wird die DBT entsprechend als das Verfahren mit dem vergleichsweise höchsten Evidenzgrad geführt [17].

Studien unter realen Bedingungen. Um die Übertragbarkeit der Ergebnisse von RCTs auf reale Versorgungsbedingungen zu überprüfen, fokussieren in den letzten Jahren zunehmend mehr Studien die Wirksamkeit der DBT unter Bedingungen, die hinsichtlich Ein- und Ausschlusskriterien, Zuweisungskriterien, therapeutischen Rahmenbedingungen/Setting oder Therapeutenauswahl weniger einschränkend sind. In einer kürzlich durchgeführten Studie zur Wirksamkeit der DBT unter realen Versorgungsbedingungen (Berliner BorderlineVersorgungsstudie, BBV) konnte gezeigt werden, dass die Ergebnisse hinsichtlich Effektstärken, Therapiedosis, Schwere der Störung und DBT-Adhärenz mit denen von RCTs vergleichbar sind [18]. So zeigten die PräPost-Ergebnisse über ein Jahr eine deutliche Reduktion des SVV, der Anzahl der stationären Aufenthalte (durchschnittlich 51 Tage in den 12 Monaten vor Behandlungsbeginn versus durchschnittlich 7 Tage während des Behandlungsjahrs), der Schwere der Borderline-Symptomatik und der allgemeinen Psychopathologie. $77 \%$ der Patienten erfüllten nach einem Jahr nicht mehr die Kriterien einer BPS nach DSM-IV-R [19]. Die Drop-out-Rate betrug 32,9\%. Darüber hinaus konnte gezeigt werden, dass es bei Therapeuten, die zeitgleich an einem Konsultationsteam teilgenommen haben, zu signifikant weniger Therapieabbrüchen kam.

Reduzierte Krankheitskosten. Zusätzlich zur verbesserten Pathologie der Patienten reduzieren sich bei Durchführung einer DBT die Krankheitskosten deutlich. In der BBV-Studie konnten die Gesamtkosten (direkte wie auch indirekte Kosten) um $9268 €$ auf $18758 €$ pro Patient im ersten Behandlungsjahr mit DBT reduziert werden (direkte Kosten: 10542€, indirekte Kosten $8234 €$ ) [20]. Im ersten Follow-up-Jahr reduzierten sich die Kosten weiter auf insgesamt $14750 €$ (direkte Kosten: $6549 €$, indirekte Kosten $8201 €$ ). Die Einsparung bei den vollstationären bzw. teilstationären Ausgaben beläuft sich auf $82 \%$ (14167€ vs. 1719€); gleichzeitig erhöhen sich allerdings die Kosten für die ambulante Therapie von $895 €$ in den 12 Monaten vor Behandlungsbeginn (keine DBT) auf 5532€ im ersten Behandlungsjahr (DBT), im Follow-up-Zeitraum auf $2632 €($ DBT).

\section{Modifikationen in der DBT}

Aufgrund ihres modularen Aufbaus hat die DBT insbesondere in den vergangenen Jahren weitreichende Modifikationen erfahren. Diese Modifikationen betreffen sowohl die Behandlung anderer oder komorbider Störungen wie auch die Behandlung in unterschiedlichen Settings. Beispiele hierfür sind:

- DBT bei komorbider Substanzabhängigkeit (DBT-S) [21]

- DBT bei Essstörungen [22]

- DBT bei ADHS [23]

- DBT bei PTBS (DBT-PTSD) [5]

- DBT bei Störungen im Jugendalter (DBT-A) [24]

- DBT im stationären Setting [25]

Praxisnahe Literatur. Die praxisnahe Anwendung der DBT in ihren unterschiedlichen Modifikationen kann in dem „Fallbuch DBT“ von Stiglmayr und Leihener nachgelesen werden [26]. Die manualisierte Anwendung der DBT im ambulanten Bereich wird in einem aktuellen Buch von Stiglmayr und Gunia ausführlich beschrieben [9].

KERNAUSSAGEN

Personen mit einer Borderline-Persönlichkeitsstörung (BPS) können ihre Emotionen nicht adäquat regulieren. Sie reagieren zunächst mit einem Vermeidungsverhalten gegenüber den unsicher empfundenen Emotionen und zeigen zur Kompensation langfristig z. T. sehr (selbst) schädigende Verhaltensweisen inklusive Suizidalität. Die Dialektisch-Behaviorale Therapie gehört zur sog. „dritten Welle“ der Verhaltenstherapien und stellt den Prototyp dar für modular aufgebaute Psychotherapie. Neben den etablierten kognitiv-behavioralen Methoden integriert die DBT eine Vielzahl weiterer Strategien und Techniken. Diese helfen dem Patienten, funktionale Strategien zur Emotionsregulation aufzubauen bzw. zu verbessern und gleichzeitig schweres Problemverhalten zu verringern, wie z. B. suizidales oder selbstschädigendes Verhalten. Ist dies gelungen, behandelt die DBT auch weitere Problemfelder wie soziale Isolation, Arbeitslosigkeit, andere komorbide psychische Störungen (u.a. posttraumatische Belastungsstörung oder Essstörungen). Neben der Borderline-Störung wird die DBT als Therapieverfahren mittlerweile bei zahlreichen weiteren Störungsbildern eingesetzt.

Interessenkonflikt

Der Autor gibt an, dass kein Interessenkonflikt besteht. 


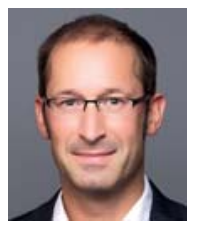

\section{Christian Stiglmayr}

Priv.-Doz. Dr. Christian Stiglmayr. Jahrgang 1964. Nach dem Studium der Psychologie von 1987-1994 wissenschaftlicher Mitarbeiter an der Psychiatrischen Universitätsklinik Freiburg. Stationspsychologe der ersten DBTStation in Deutschland. 2002 Promotion. 2001-2004 wissenschaftlicher Mitarbeiter an der FU Berlin. 2001 Gründung und seitdem Leiter der AWP-Berlin. Seit 2002 niedergelassen als Psychologischer Psychotherapeut (VT) in Berlin. 2010 Habilitation und seitdem Privatdozent an der HU zu Berlin. Dozent, Trainer und Supervisor für DBT und VT.

\section{Korrespondenzadresse}

PD Dr. Christian Stiglmayr

Arbeitsgemeinschaft für Wissenschaftliche

Psychotherapie (AWP-Berlin)

Witzlebenstraße 30A

14057 Berlin

E-Mail: christian.stiglmayr@awp-berlin.de

Verantwortlicher Herausgeber für diesen Beitrag

Prof. Dr. Klaus Lieb, Mainz

Literatur

[1] Linehan MM. Dialektisch-Behaviorale Therapie der BPS. München: CIP; 1996 a

[2] Linehan MM. Trainingsmanual der Dialektisch-Behavioralen Therapie der BPS. München: CIP; 1996 b

[3] Stiglmayr CE. Grathwol T. Linehan MM et al. Aversive tension in patients with borderline personality disorder: A computer-based controlled field study. Acta Psychiatrica Scand 2005; 111: $372-379$

[4] Santangelo P. Reinhard I. Mussgay L et al. Specificity of affective instability in patients with borderline personality disorder compared to posttraumatic stress disorder, bulimia nervosa, and healthy controls. J Abnormal Psychol 2014; 123: 258-272

[5] Bohus M. Dyer A. Priebe K et al. Dialectical behaviour therapy for post-traumatic stress disorder after childhood sexual abuse in patients with and without borderline personality disorder: a randomised controlled trial. Psychother Psychosom 2013; 82: 221-233

[6] Potreck-Rose F. Jacob G. Selbstzuwendung, Selbstakzeptanz und Selbstvertrauen. Psychotherapeutische Interventionen zum Aufbau von Selbstwertgefühl. 9. Aufl. Stuttgart: Pfeiffer bei Klett-Cotta; 2015

[7] Germer C. Der achtsame Weg zur Selbstliebe. Freiburg: Arbor; 2012

[8] Gilbert P. Compassion Focused Therapy. Paderborn: Junfermann; 2013

[9] Stiglmayr CE. Gunia H. Dialektisch-Behaviorale Therapie (DBT) zur Behandlung der Borderline-Persönlichkeisstö- rung. Ein Manual für die ambulante Therapie. Göttingen: Hogrefe; 2017

[10] Bohus M. Wolf M. Interaktives Skills-Training für BorderlinePatienten. 2. Auflage. Stuttgart: Schattauer Verlag; 2013

[11] Gunderson JG. Frank AF. Ronningstam EF et al. Early discontinuance of borderline patients from psychotherapy. J Nervous Mental Dis 1989; 177: 38-42

[12] Arntz A. van Gendersen H. Schematherapie bei BorderlinePersönlichkeitsstörung. Weinheim: Beltz; 2010

[13] Bateman A. Fonagy P. Mentalization-Based Treatment for Borderline Personality Disorder. A practical Guide. Oxford: Oxford University Press; 2006

[14] Clarkin JF. Yeomans FE. Kernberg OF. Psychotherapie der Borderline Persönlichkeit. Manual zur psychodynamischen Therapie. Stuttgart: Schattauer; 2001

[15] McMain SF. Links PS. Gnam WH et al. A randomized trial of dialectical behavior therapy versus general psychiatric management for borderline personality disorder. Am J Psychiatry 2009; 166: 1365-1374

[16] Stoffers JM. Völlm BA. Rücker G et al. Psychological therapies for people with borderline personality disorder. Cochrane Database of Systematic Reviews 2012; 8: CD005652

[17] Deutsche Gesellschaft für Psychiatrie Psychotherapie und Nervenheilkunde (DGPPN). Leitlinien für Persönlichkeitsstörungen. Heidelberg: Steinkopff-Verlag; 2009

[18] Stiglmayr CE. Stecher-Mohr J. Wagner T et al. Effectiveness of dialectical behavioral therapy in routine outpatient care: The Berlin Borderline Study. Borderline Personality Disorder and Emotion Dysregulation 2015; 1: 20

[19] American Psychiatric Association. Diagnostisches und Statistisches Manual Psychischer Störungen - Textrevision DSM-IV-TR. (P. Falkai u. H.-U. Wittchen, deutsche Ausgabe). Göttingen: Hogrefe; 2003

[20] Wagner T. Fydrich T. Stiglmayr CE et al. Societal cost-of-illness in patients with borderline personality disorder one year before, during and after dialectical behavior therapy in routine outpatient care. Behav Res Ther 2014; 61: 12-22

[21] Daig I. Bermpohl F. Kienast T. Psychotherapie von Patienten mit Borderline Persönlichkeitsstörung und Abhängigkeitserkrankung. Sucht 2011; 57: 363-371

[22] Sipos V. Bohus M. Schweiger U. Dialektisch-Behaviorale Therapie für Essstörungen (DBT-E). Psychother Psychosom medizinische Psychologie 2011; 61: 87-91

[23] Hesslinger B. Philipsen A. Richter H. Psychotherapie der ADHS im Erwachsenenalter. Ein Arbeitsbuch. Göttingen: Hogrefe; 2004

[24] Fleischhaker C. Sixt B. Schulz E. DBT-A. Dialektisch-Behaviorale Therapie für Jugendliche. Berlin: Springer; 2010

[25] Bohus M. Haaf B. Simms T et al. Effectiveness of inpatient dialectical behavioral therapy for borderline personality disorder: a controlled trial. Behav Res Ther 2004; 42: 487 499

[26] Stiglmayr CE. Leihener F, Hrsg. Fallbuch DBT. Weinheim: Beltz; 2015

Bibliografie

DOI http://dx.doi.org/10.1055/s-0042-123718

PSYCH up2date 2017; 11: 151-164

(c) Georg Thieme Verlag KG Stuttgart · New York ISSN 2194-8895 


\section{Punkte sammeln auf CME.thieme.de}

Diese Fortbildungseinheit ist 12 Monate online für die Teilnahme verfügbar.

Sollten Sie Fragen zur Online-Teilnahme haben, finden Sie unter http://cme.thieme.de/hilfe eine ausführliche Anleitung. Wir wünschen viel Erfolg beim Beantworten

der Fragen!

Unter https://eref.thieme.de/ZZWHQ6V oder über den QR-Code kommen Sie direkt zum Artikel zur Eingabe der Antworten.

VNR 2760512017152374452

\section{Frage 1}

Welche Aussage trifft zu? Im Verständnis der DBT handelt es sich bei der Borderline-Persönlichkeitsstörung um eine...

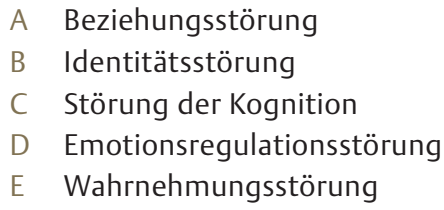

\section{Frage 2}

\section{Auf welchen 2 Säulen ruht die DBT?}

A Die eine Säule symbolisiert die zur Anwendung kommenden Therapiestruktur; die andere Säule symbolisiert die Haltung des Therapeuten.

B Die eine Säule symbolisiert die zur Anwendung kommenden Techniken/Strategien; die andere Säule symbolisiert die Einzeltherapie.

C Die eine Säule symbolisiert die zur Anwendung kommenden Techniken/Strategien; die andere Säule symbolisiert die Haltung des Therapeuten.

D Die eine Säule symbolisiert das zur Anwendung kommende Fertigkeitentraining; die andere Säule symbolisiert die Haltung des Therapeuten.

E Die eine Säule symbolisiert die zur Anwendung kommenden veränderungsorientierten Strategien; die andere Säule symbolisiert die akzeptanzorientierten Strategien.

\section{Frage 3}

Wie viele Phasen werden im Ablauf einer DBT unterschieden (inklusive Vorbereitungsphasen)?
A 1
B 2
C 3
D 4
E 5

\section{Frage 4}

Nach welchem Kriterium ist die Behandlungshierarchie in der ersten Therapiephase aufgebaut?

A Die Behandlungshierarchie orientiert sich an den Wünschen des Patienten.

B Die Behandlungshierarchie orientiert sich an der Gefährlichkeit des gezeigten Verhaltens.

C Die Behandlungshierarchie orientiert sich an der Schwere der Komorbiditäten.

D Die Behandlungshierarchie orientiert sich an der Schwere der zugrunde liegenden Traumata.

E Die Behandlungshierarchie orientiert sich an der Schwere der zwischenmenschlichen Schwierigkeiten.

\section{Frage 5}

Aus welchen Therapiebausteinen besteht die DBT?

A Einzeltherapie, Skillstraining, Supervision, Krisenintervention

B Einzeltherapie, Skillstraining, Telefonkontakte, Konsultationsteam

C Einzeltherapie, Skillstraining, Telefonkontakte, Konsultationsteam, Supervision, Krisenintervention

D Einzeltherapie, Skillstraining, Telefonkontakte, Konsultationsteam, Supervision

E Einzeltherapie, Skillstraining, Telefonkontakte, Supervision, Krisenintervention 


\section{CME-Fragen bei CME.thieme.de}

Fortsetzung ...

\section{Frage 6}

Welche Module werden im Fertigkeitentraining vermittelt?

A Stresstoleranz, Umgang mit Gefühlen, zwischenmenschliche Fertigkeiten, Achtsamkeit, Umgang mit Sucht, Steigerung des Selbstwerts

B Stresstoleranz, Umgang mit Gefühlen, zwischenmenschliche Fertigkeiten, Achtsamkeit, Umgang mit Sucht, Steigerung des Selbstmitgefühls

C Stresstoleranz, Umgang mit Gefühlen, kognitive Fertigkeiten, Achtsamkeit, Umgang mit Sucht, Steigerung des Selbstwerts

D Stresstoleranz, Umgang mit Gefühlen, zwischenmenschliche Fertigkeiten, radikale Akzeptanz, Umgang mit Sucht, Steigerung des Selbstwerts

E Stresstoleranz, Umgang mit Gefühlen, zwischenmenschliche Fertigkeiten, Achtsamkeit, Umgang mit Sucht, Umgang mit Traumata

\section{Frage 7}

Wie viele Grundannahmen gibt es in der DBT?
A 2
B 4
C 6
D 8
E 10

\section{Frage 8}

Was sind die 2 wesentlichen Strategienblöcke, die in der DBT zur Anwendung kommen?

A dialektische Strategien vs. strukturelle Strategien

B Problemlösestrategien vs. Validierungsstrategien

C akzeptanzbasierte Strategien vs. strukturelle Strategien

D Validierungsstrategien vs. veränderungsorientierte Strategien

E akzeptanzbasierte Strategien vs. veränderungsorientierte Strategien

\section{Frage 9}

Welche Aussage trifft zu?

A In der DBT wird dysfunktionales Verhalten niemals über die Beziehung verstärkt, funktionales Verhalten hingegen immer.

B In der DBT wird sowohl funktionales wie auch dysfunktionales Verhalten niemals über einen Beziehungsentzug bestraft.

C In der DBT wird dysfunktionales Verhalten niemals ignoriert, funktionales Verhalten hingegen immer.

D In der DBT wird dysfunktionales Verhalten immer besprochen, funktionales Verhalten nur wenn nötig.

E In der DBT wird dysfunktionales Verhalten genauso behandelt wie funktionales Verhalten.

\section{Frage 10}

Was besagt die Metapher der Kinderwippe („Teeter-Totter“) hinsichtlich der Beziehungsgestaltung?

A Bewegt sich der Patient weg von dem Therapeuten, geht dieser hinterher.

B Droht der Patient über die Mittelachse auf die Seite des Therapeuten zu kommen, geht der Therapeut weg.

C Bewegt sich der Patient weg von dem Therapeuten, geht dieser ebenfalls nach hinten.

D Kommt der Patient auf den Therapeuten zu, bewegt sich dieser weg.

E Verlässt der Patient die Wippe, bleibt der Therapeut auf dieser stehen. 